\title{
Modeling MEK4 Kinase Inhibitors Through Perturbed Electrostatic Potential Charges
}

Rama K. Mishra*1,2, Kristine K. Deibler ${ }^{3}$, Matthew R. Clutter ${ }^{4,5}$, Purav P. Vagadia ${ }^{1}$, Matthew

O'Connor', Gary E. Schiltz ${ }^{1,2,5}$, Raymond Bergan ${ }^{6}$ and Karl A. Scheidt ${ }^{* 1,2,3,4,5}$

${ }^{1}$ Center for Molecular Innovation and Drug Discovery, Northwestern University, 2145 Sheridan Rd, Evanston, Illinois 60208, United States

${ }^{2}$ Department of Pharmacology, Feinberg School of Medicine, Northwestern University, Chicago, Illinois 60611, United States

${ }^{3}$ Department of Chemistry, Northwestern University, Evanston, 60208, Illinois, United States

${ }^{4}$ Chemistry of Life Processes Institute, Northwestern University, 2145 Sheridan Rd, Evanston, Illinois 60208, United States

${ }^{5}$ Robert H. Lurie Comprehensive Cancer Center, Feinberg School of Medicine, Northwestern University, Chicago, IL 60611 (USA)

${ }^{6}$ Knight Cancer Institute, Oregon Health \& Science University, Portland, OR 97239 (USA)

Corresponding Authors: Rama K. Mishra; Karl A. Scheidt

E-mail: r-mishra@northwestern.edu; scheidt@northwestern.edu 


\section{SI Tables}

Table S1. Quinoline set observed $\mathrm{IC}_{50}$, Hydrogen Bond Energy ( $\left.\mathrm{kcal} / \mathrm{mol}\right)$ and perturbed ESP charges of donor (D) and acceptor (A) atoms of ligand and hinge residues in esu units.

\begin{tabular}{|c|c|c|c|c|c|c|c|}
\hline Structure & $\begin{array}{l}\text { Name } \\
\text { NUCC \# }\end{array}$ & $I C_{50}(\mu M)$ & $\begin{array}{l}\text { HB- } \\
\text { Energy }\end{array}$ & $\Delta A$ Lig & $\Delta D$ Lig & $\Delta A$ Hinge & $\Delta D$ Hinge \\
\hline & 0181794 & 0.57 & -6.57 & 0.47 & 0.0 & 0.09 & 0.28 \\
\hline & 0181589 & 0.84 & -5.68 & 0.38 & 0.0 & 0.08 & 0.2 \\
\hline & 0181792 & 0.96 & -4.88 & 0.33 & 0.0 & 0.08 & 0.02 \\
\hline & 0181669 & 1.1 & -6.94 & 0.39 & 0.0 & 0.06 & 0.22 \\
\hline & 0181791 & 2.1 & -4.96 & 0.35 & 0.0 & 0.6 & 0.18 \\
\hline & 0185593 & 3.2 & -1.55 & 0.33 & 0.0 & 0.01 & 0.22 \\
\hline & 0181703 & 4.0 & -6.10 & 0.34 & 0.0 & 0.09 & 0.21 \\
\hline
\end{tabular}




\begin{tabular}{|l|l|l|l|l|l|l|} 
& & & & 0.25 \\
\hline & & & & & & \\
\end{tabular}


Table S2. Indazole set observed $\mathrm{IC}_{50}$, Hydrogen Bond Energy $(\mathrm{kcal} / \mathrm{mol})$ and perturbed ESP charges of donor (D) and acceptor (A) atoms of ligand and hinge residues in esu units.

\begin{tabular}{|c|c|c|c|c|c|c|c|}
\hline Structure & $\begin{array}{l}\text { Name } \\
\text { NUCC\# }\end{array}$ & $I C_{50}(\mu M)$ & $\begin{array}{l}\text { HB- } \\
\text { Energy }\end{array}$ & $\Delta A$ Lig & $\Delta D$ Lig & $\Delta A$ Hinge & $\Delta D$ Hinge \\
\hline & 0201177 & 0.041 & -7.03 & 0.29 & 0.01 & 0.49 & 0.10 \\
\hline & 0201167 & 0.047 & -10.55 & 0.29 & 0.13 & 0.51 & 0.12 \\
\hline & 0201178 & 0.12 & -8.03 & 0.28 & 0.04 & 0.51 & 0.21 \\
\hline & 0200886 & 0.14 & -7.97 & 0.27 & 0.003 & 0.54 & 0.14 \\
\hline & 0201174 & 0.42 & -10.54 & 0.27 & 0.04 & 0.53 & 0.19 \\
\hline & 0201169 & 0.45 & -10.91 & 0.26 & 0.01 & 0.55 & 0.07 \\
\hline & 0200883 & 0.45 & -7.33 & 0.27 & 0.02 & 0.54 & 0.25 \\
\hline & 0201179 & 0.47 & -6.81 & 0.29 & 0.01 & 0.49 & 0.10 \\
\hline
\end{tabular}




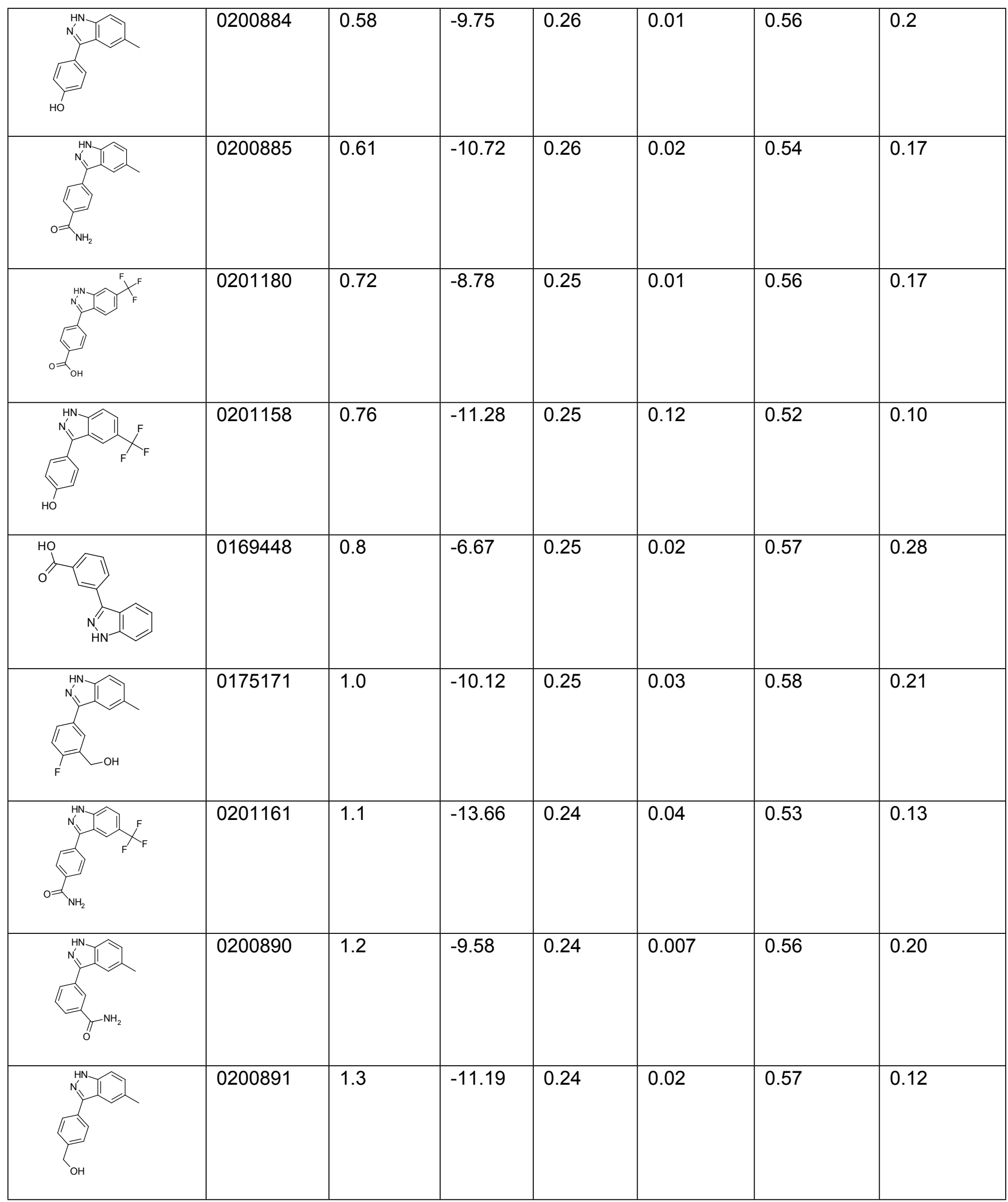




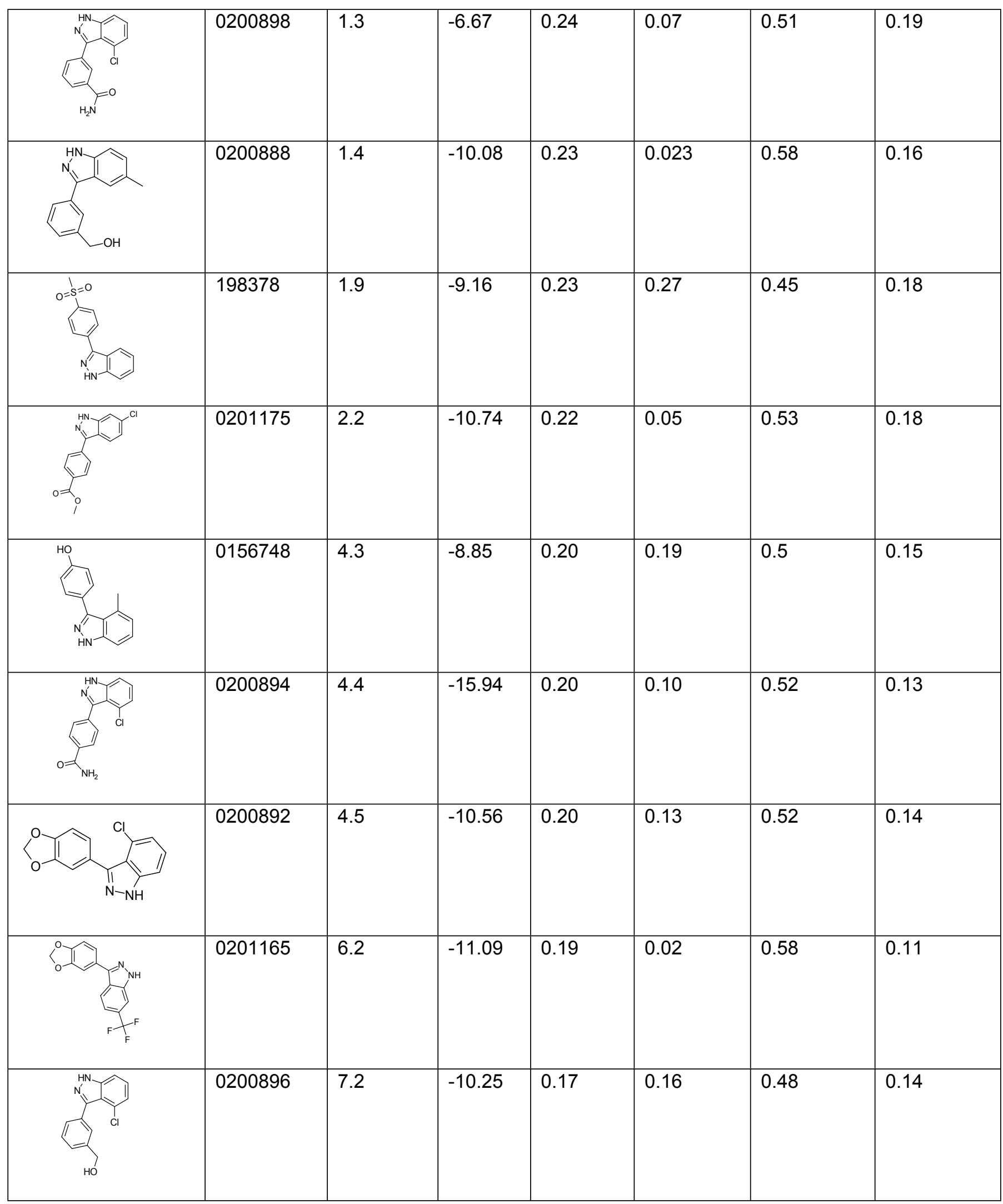




\begin{tabular}{|c|c|c|c|c|c|c|c|}
\hline & 0200899 & 8.1 & -12.35 & 0.16 & 0.05 & 0.54 & 0.16 \\
\hline & 0198379 & 12.0 & -9.78 & 0.17 & 0.22 & 0.46 & 0.11 \\
\hline & 0198376 & 15.0 & -8.0 & 0.09 & 0.03 & 0.47 & 0.25 \\
\hline & 0200889 & 17.0 & -6.58 & 0.10 & 0.07 & 0.48 & 0.22 \\
\hline & 198377 & 130.0 & -5.33 & 0.03 & 0.05 & 0.56 & 0.25 \\
\hline
\end{tabular}


Table S3: Thiazole set observed $\mathrm{IC}_{50}$, Hydrogen Bond Energy $(\mathrm{kcal} / \mathrm{mol})$ perturbed ESP charges of donor(D) and acceptor(A) atoms of ligand and hinge residues in esu units

\begin{tabular}{|c|c|c|c|c|c|c|c|}
\hline Structure & $\begin{array}{l}\text { Name } \\
\text { NUCC\# }\end{array}$ & $I_{50}(\mu \mathrm{M})$ & $\begin{array}{l}\text { HB- } \\
\text { Energy }\end{array}$ & $\Delta A \operatorname{Lig}$ & $\Delta D$ Lig & $\Delta A$ Hinge & $\Delta D$ Hinge \\
\hline & 0176798 & 0.78 & -25.37 & 0.27 & 0.02 & 0.17 & 0.24 \\
\hline & 0185360 & 0.94 & -25.46 & 0.26 & 0.01 & 0.18 & 0.13 \\
\hline & 0176863 & 1 & -24.79 & 0.25 & 0.07 & 0.17 & 0.12 \\
\hline & 0176871 & 1.1 & -27.36 & 0.24 & 0.09 & 0.15 & 0.25 \\
\hline & 0176869 & 1.2 & -20.58 & 0.23 & 0.1 & 0.18 & 0.19 \\
\hline & 0180108 & 1.2 & -26.67 & 0.22 & 0.03 & 0.16 & 0.22 \\
\hline
\end{tabular}




\begin{tabular}{|c|c|c|c|c|c|c|c|}
\hline & 0176770 & 1.3 & -22.55 & 0.22 & 0.01 & 0.05 & 0.15 \\
\hline & 0188171 & 1.6 & -18.65 & 0.22 & 0.03 & 0.09 & 0.1 \\
\hline & 0176877 & 1.7 & -27.29 & 0.23 & 0.08 & 0.14 & 0.24 \\
\hline & 0176875 & 1.7 & -23.42 & 0.21 & 0.06 & 0.18 & 0.25 \\
\hline & 0176870 & 1.7 & -12.39 & 0.2 & 0.03 & 0.16 & 0.27 \\
\hline & 0176873 & 2 & -25.67 & 0.21 & 0.13 & 0.2 & 0.23 \\
\hline & 0176879 & 2 & -6.39 & 0.22 & 0.01 & 0.14 & 0.19 \\
\hline & 0176415 & 2.3 & -26.80 & 0.20 & 0.05 & 0.17 & 0.25 \\
\hline
\end{tabular}




\begin{tabular}{|c|c|c|c|c|c|c|c|}
\hline & 0176835 & 2.9 & -18.71 & 0.21 & 0.02 & 0.16 & 0.09 \\
\hline & 0176874 & 3 & -16.45 & 0.17 & 0.06 & 0.19 & 0.3 \\
\hline & 0176757 & 3.1 & -13.38 & 0.16 & 0.04 & 0.15 & 0.19 \\
\hline & 0180102 & 3.3 & -10.28 & 0.17 & 0.22 & 0.16 & 0.36 \\
\hline & 0176809 & 3.7 & -23.57 & 0.18 & 0.01 & 0.17 & 0.29 \\
\hline & 0176776 & 3.9 & -25.88 & 0.19 & 0.03 & 0.19 & 0.26 \\
\hline & 0188675 & 4.8 & -28.30 & 0.17 & 0.03 & 0.15 & 0.24 \\
\hline & 0180086 & 5.3 & -11.24 & 0.17 & 0.06 & 0.16 & 0.18 \\
\hline
\end{tabular}




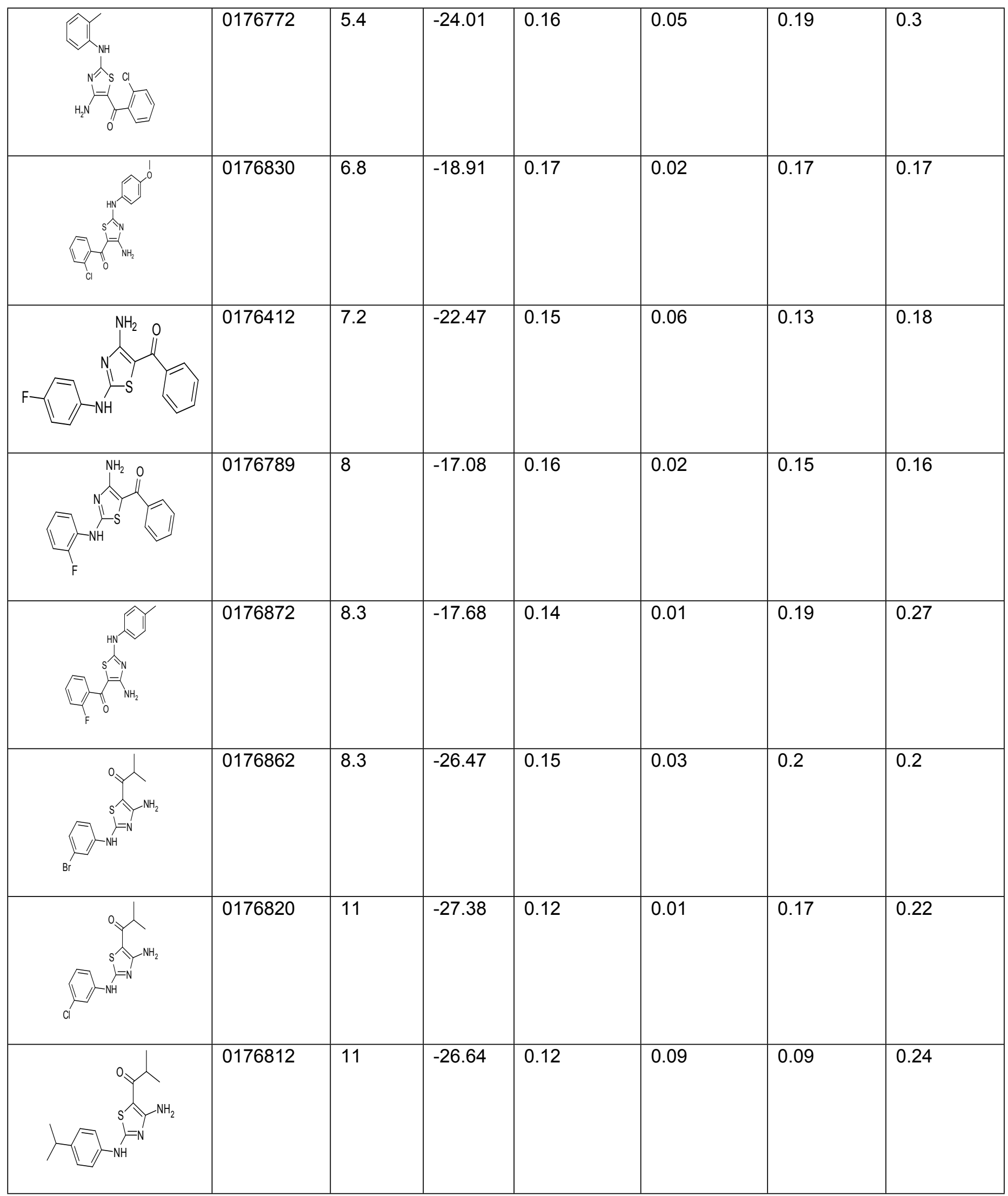




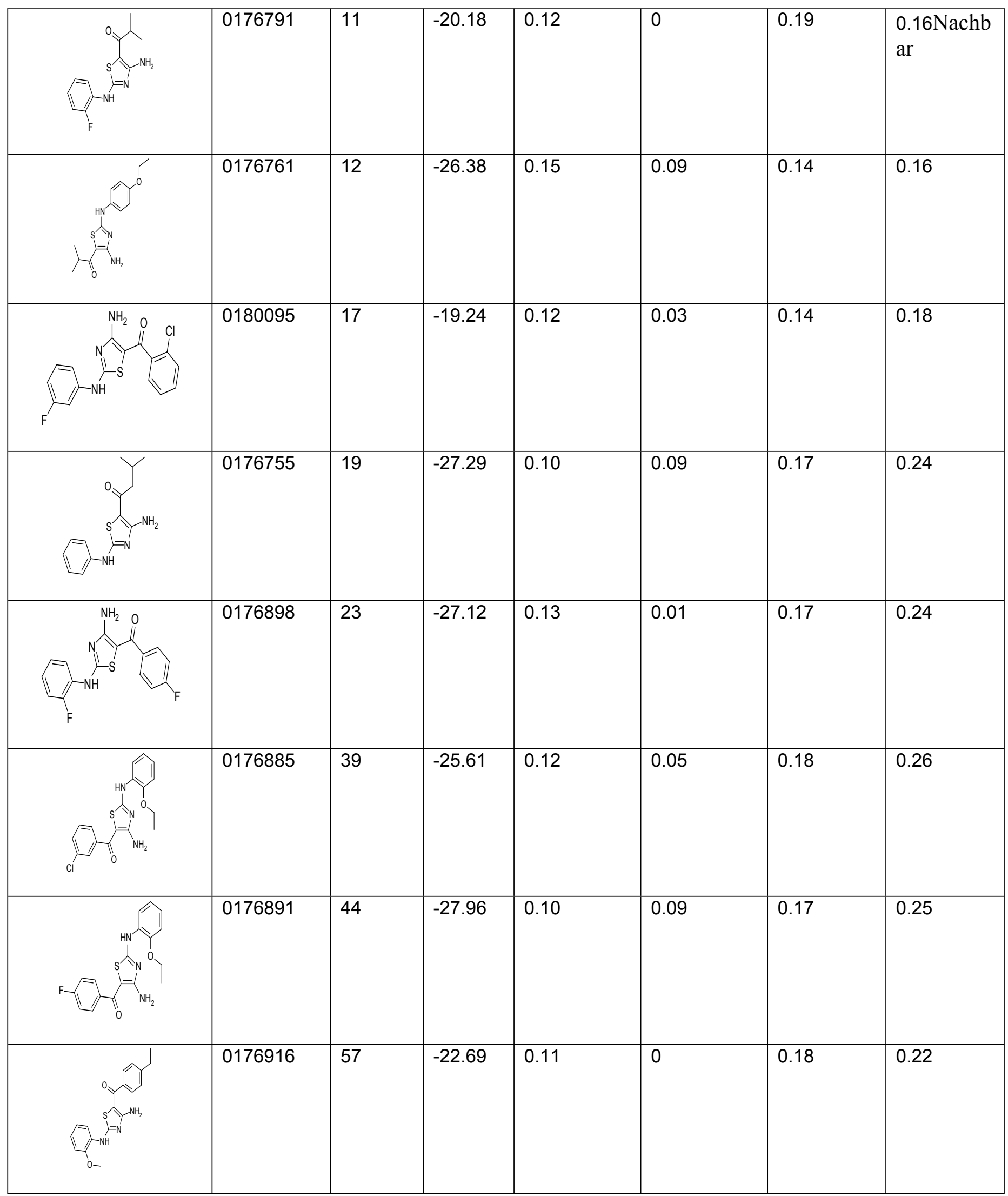


Table S4: Values of observed and predicted IC50, perturbed ESP charges in esu units, cLogP and PSA.

\begin{tabular}{|c|c|c|c|c|c|c|c|c|c|}
\hline Structure & Name & $\begin{array}{l}I_{50} \\
\text { Obs }\end{array}$ & $\begin{array}{c}I C_{50} \\
\text { Predict }\end{array}$ & $\begin{array}{l}\Delta A \\
L i g\end{array}$ & $\begin{array}{l}\Delta D \\
L i g\end{array}$ & $\begin{array}{c}\Delta A \\
\text { Hinge }\end{array}$ & $\begin{array}{c}\Delta D \\
\text { Hinge }\end{array}$ & cLogP & $P S A$ \\
\hline & $\begin{array}{c}\text { NUCC- } \\
0181703\end{array}$ & 4.0 & 3.74 & 0.34 & 0.0 & 0.09 & 0.21 & 5.74 & 97.82 \\
\hline & $\begin{array}{c}\text { NUCC- } \\
0181668\end{array}$ & 5.8 & 4.2 & 0.38 & 0.0 & 0.05 & 0.23 & 4.43 & 90.44 \\
\hline & $\begin{array}{l}\text { NUCC- } \\
0181588\end{array}$ & 6.5 & 7.81 & 0.30 & 0.0 & 0.07 & 0.10 & 4.40 & 90.45 \\
\hline & $\begin{array}{l}\text { NUCC- } \\
0185592\end{array}$ & 9.3 & 9.45 & 0.31 & 0.0 & 0.07 & 0.22 & 3.69 & 90.44 \\
\hline & $\begin{array}{c}\text { NUCC- } \\
0181793\end{array}$ & 13.0 & 8.78 & 0.28 & 0.0 & 0.07 & 0.20 & 5.19 & 96.16 \\
\hline & $\begin{array}{l}\text { NUCC- } \\
0201158\end{array}$ & 0.76 & 0.39 & 0.25 & 0.13 & 0.52 & 0.10 & 4.41 & 113.72 \\
\hline & $\begin{array}{c}\text { NUCC- } \\
0198378\end{array}$ & 1.9 & 0.89 & 0.23 & 0.27 & 0.46 & 0.18 & 2.27 & 131.31 \\
\hline & $\begin{array}{l}\text { NUCC- } \\
0156748\end{array}$ & 4.3 & 3.39 & 0.20 & 0.19 & 0.50 & 0.15 & 3.74 & 117.54 \\
\hline & $\begin{array}{c}\text { NUCC- } \\
0200894\end{array}$ & 4.4 & 2.90 & 0.20 & 0.11 & 0.52 & 0.13 & 3.24 & 152.61 \\
\hline & $\begin{array}{c}\text { NUCC- } \\
0200892\end{array}$ & 4.5 & 5.41 & 0.19 & 0.13 & 0.51 & 0.14 & 4.57 & 98.80 \\
\hline
\end{tabular}




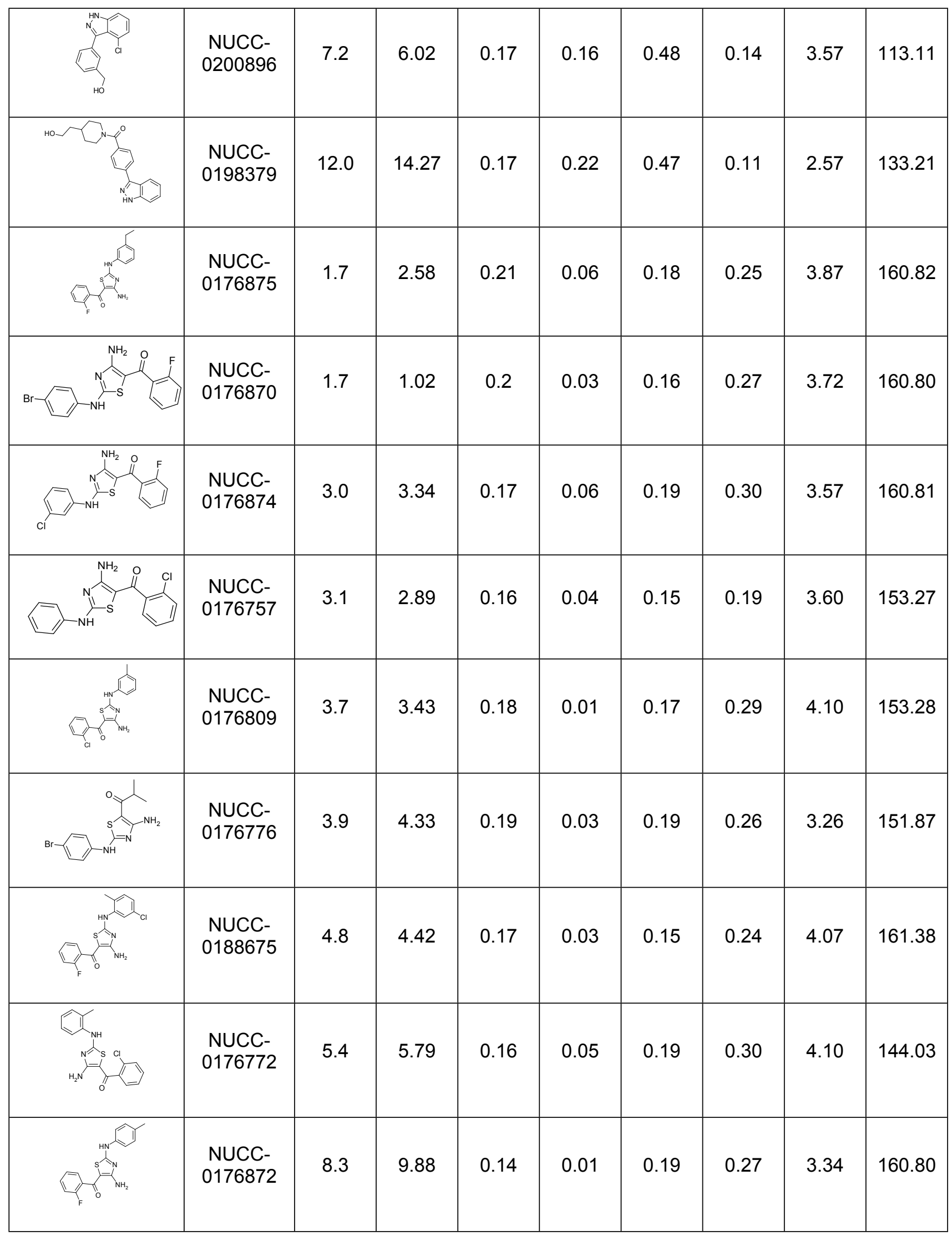




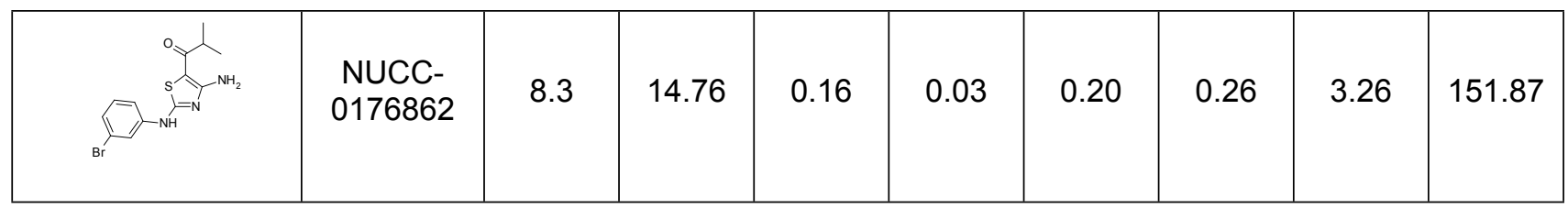

Table S5: The inter-correlation heat map of the 4 new descriptors used in MLR

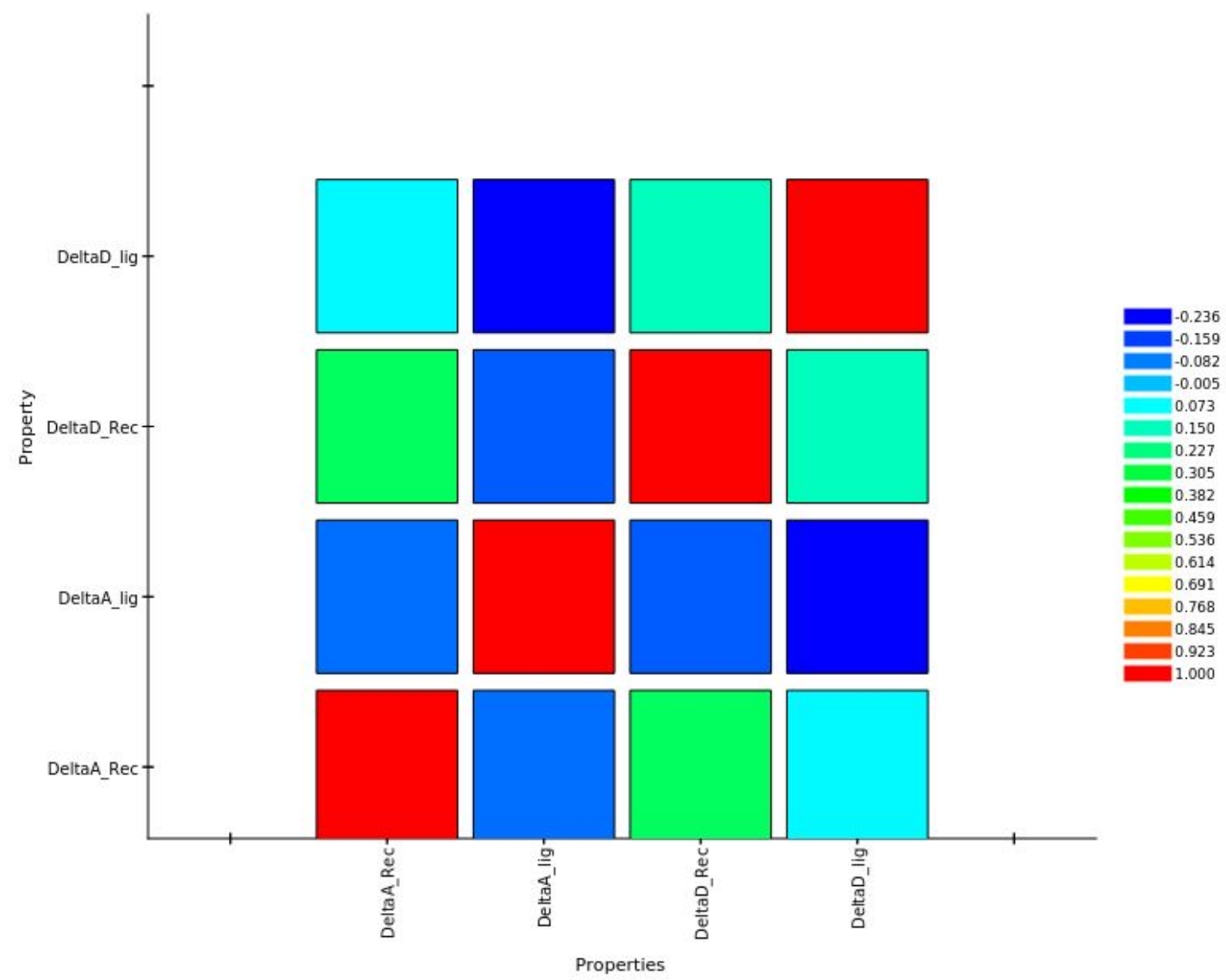


Table S6: The training set domain applicability data

\begin{tabular}{|l|l|l|l|l|}
\hline Variable & Min & Max & Mean & St. Dev \\
\hline pIC50 & 3.89 & 7.39 & 5.60 & 0.73 \\
\hline$\Delta$ D_Rec & 0.07 & 0.36 & 0.19 & 0.06 \\
\hline$\Delta$ A_Rec & -0.58 & 0.60 & --0.12 & 0.34 \\
\hline$\Delta$ D_Lig & 0.00 & 0.22 & 0.04 & 0.04 \\
\hline$\Delta$ A_Lig & 0.03 & & 0.22 & 0.08 \\
\hline
\end{tabular}

